\section{OPEN ACCESS}

Edited by:

Miklos Fuzi,

Semmelweis University, Hungary

Reviewed by:

Luca A. Vitali,

University of Camerino, Italy

Zhi Ruan,

Sir Run Run Shaw Hospital, China

*Correspondence:

Mei Zeng

zengmeigao@aliyun.com

Mingliang Chen

chenmingliang@scdc.sh.cn

tThese authors have contributed equally to this work.

Specialty section:

This article was submitted to Antimicrobials, Resistance and

Chemotherapy,

a section of the journal

Frontiers in Microbiology

Received: 20 November 2017 Accepted: 09 March 2018

Published: 23 March 2018

Citation:

Shen Y, Cai J, Davies MR, Zhang C, Gao K, Qiao D, Jiang H, Yao W, Li Y,

Zeng $M$ and Chen $M$ (2018)

Identification and Characterization of

Fluoroquinolone Non-susceptible

Streptococcus pyogenes Clones Harboring Tetracycline and Macrolide

Resistance in Shanghai, China.

Front. Microbiol. 9:542.

doi: 10.3389/fmicb.2018.00542

\title{
Identification and Characterization of Fluoroquinolone Non-susceptible Streptococcus pyogenes Clones Harboring Tetracycline and Macrolide Resistance in Shanghai, China
}

\begin{abstract}
Yinfang Shen ${ }^{1,2+}$, Jiehao Cai ${ }^{1+}$, Mark R. Davies ${ }^{3}$, Chi Zhang ${ }^{4}$, Kun Gao ${ }^{5}$, Dan Qiao ${ }^{6}$, Haoqin Jiang ${ }^{7}$, Weilei Yao ${ }^{1}$, Yuefang $\mathrm{Li}^{1}$, Mei Zeng ${ }^{1 *}$ and Mingliang Chen ${ }^{4,8 *}$

${ }^{1}$ Department of Infectious Diseases, Children's Hospital of Fudan University, Shanghai, China, ${ }^{2}$ Department of Pediatrics, Jinshan Hospital, Fudan University, Shanghai, China, ${ }^{3}$ Department of Microbiology and Immunology, Peter Doherty Institute for Infection and Immunity, University of Melbourne, Melbourne, VIC, Australia, ${ }^{4}$ Department of Microbiology, Shanghai Municipal Center for Disease Control and Prevention, Shanghai, China, ${ }^{5}$ Department of Clinical Laboratory, Xuhui Dahua Hospital, Shanghai, China, ${ }^{6}$ Department of Clinical Laboratory, Ruijin Hospital (North), Shanghai Jiaotong University School of Medicine, Shanghai, China, ' Department of Laboratory Medicine, Shanghai Medical College, Huashan Hospital, Fudan University, Shanghai, China, ${ }^{8}$ Department of Microbiology, Shanghai Institutes of Preventive Medicine, Shanghai, China
\end{abstract}

Streptococcus pyogenes, also known as group A Streptococcus (GAS), is one of the top 10 infectious causes of death worldwide. Macrolide and tetracycline resistant GAS has emerged as a major health concern in China coinciding with an ongoing scarlet fever epidemic. Furthermore, increasing rates of fluoroquinolone (FQ) non-susceptibility within GAS from geographical regions outside of China has also been reported. Fluoroquinolones are the third most commonly prescribed antibiotic in China and is an therapeutic alternative for multi-drug resistant GAS. The purpose of this study was to investigate the epidemiological and molecular features of GAS fluoroquinolone (FQ) non-susceptibility in Shanghai, China. GAS $(n=2,258)$ recovered between 2011 and 2016 from children and adults were tested for FQ-non-susceptibility. Efflux phenotype and mutations in parC, parE, gyrA, and gyrB were investigated and genetic relationships were determined by emm typing, pulsed-field gel electrophoresis and phylogenetic analysis. The frequency of GAS FQ-non-susceptibility was $1.3 \%(30 / 2,258)$, with the phenotype more prevalent in GAS isolated from adults (14.3\%) than from children (1.2\%). Eighty percent (24/30) of FQ-non-susceptible isolates were also resistant to both macrolides (ermB) and tetracycline (tetM) including the GAS sequence types emm12, emm6, emm11, and emm1. Genomic fingerprinting analysis of the 30 isolates revealed that non-susceptibility may arise in various genetic backgrounds even within a single emm type. No efflux phenotype was observed in FQ non-susceptible isolates, and molecular analysis of the quinolone resistance-determining regions (QRDRs) identified several sequence polymorphisms in ParC and ParE, and none in GyrA and GyrB. Expansion of this analysis to 152 publically available GAS whole genome sequences from Hong Kong predicted 7.9\% (12/152) of Hong Kong isolates harbored a S79F ParC mutation, of which $66.7 \%$ (8/12) were macrolide and tetracycline resistant. Phylogenetic 
analysis of the parC QRDR sequences suggested the possibility that FQ resistance may be acquired through inter-species lateral gene transfer. This study reports the emergence of macrolide, tetracycline, and fluoroquinolone multidrug-resistant clones across several GAS emm types including emm 1 and emm12, warranting continual surveillance given the extensive use of fluoroquinolones in clinical use.

Keywords: Streptococcus pyogenes, fluoroquinolone resistance, multidrug resistance, pulsed-field gel electrophoresis (PFGE), horizontal gene transfer

\section{INTRODUCTION}

Streptococcus pyogenes, ranked as one of the top 10 infectious causes of death worldwide, is responsible for more than 517,000 deaths annually (Carapetis et al., 2005). Also known as group A Streptococcus (GAS), it can cause various clinical infections, such as pharyngitis, impetigo, scarlet fever, necrotizing fasciitis, streptococcal toxic shock syndrome, and the immune mediated post-infectious manifestations of acute post-streptococcal glomerulonephritis, acute rheumatic fever and rheumatic heart disease (Walker et al., 2014). Since 2011, an unexpected pediatric scarlet fever epidemic has occurred and sustained in Hong Kong and throughout mainland China, with an incidence of 22-31 cases per 100,000 people (Chen et al., 2012; Lau et al., 2012; Tse et al., 2012; Yang et al., 2013; Davies et al., 2015). Furthermore, an ongoing scarlet fever outbreak has been reported in the United Kingdom since 2013/2014 season (Chalker et al., 2017), with an incidence of 25 cases per 100,000 people.

Penicillin and macrolides are primary antibiotic therapeutics administered for suspected S. pyogenes infections (Montes et al., 2010). Although S. pyogenes remains susceptible to penicillin, resistance to macrolides is increasing and a major cause for concern in China, with a frequency $>93 \%$ (Chen et al., 2012; Yang et al., 2013). Fluoroquinolones (FQs) are an attractive alternative when patients are hypersensitive to beta-lactam antibiotics (Montes et al., 2010). In China, consumption of FQs increased by up to $2 \times 10^{8}$ standard units during 2001-2010, becoming the third most consumed antibiotic in the adult population (Van Boeckel et al., 2014). Increasing frequencies of S. pyogenes isolates with reduced susceptibility to FQs have been observed in many countries (Reinert et al., 2004; Malhotra-Kumar et al., 2005; Smeesters et al., 2009; Montes et al., 2010; Pires et al., 2010; Van Heirstraeten et al., 2012; Petrelli et al., 2014), yet data on FQ-nonsusceptible S. pyogenes in China remain scarce despite extremely high resistance to primary interventions such as macrolides.

In Gram-positive bacteria, FQ resistance is mainly mediated through point mutations within the quinolone resistancedetermining region (QRDR) of topoisomerase IV ParC and ParE and /or the topoisomerase II DNA gyrase GyrA and GyrB and efflux pump (Hooper, 2002). In S. pyogenes, FQ resistance is reported to be conferred by either parC or gyrA (Yan et al., 2000; Albertí et al., 2005; Montes et al., 2010), and develop in a stepwise manner (Malhotra-Kumar et al., 2009). At first, mutations occurring in parC can lead to low-level resistance. Then, additional mutations in gyrA can lead to high-level resistance. Mutations in the parC and gyrA QRDRs can occur not only spontaneously but also by means of horizontal gene transfer from other streptococcal species (Pletz et al., 2006; Duesberg et al., 2008; Pinho et al., 2010).

The aim of this study was to investigate the frequency, mechanism, and epidemiological association of FQ nonsusceptibility in S. pyogenes during 2011 and 2016 from Shanghai, China.

\section{MATERIALS AND METHODS \\ Isolate Database}

In order to generate a $S$. pyogenes isolate database representative of the Shanghai population, carriage, and clinical isolates were prospectively collected from different geographical locations in Shanghai between June 2011 to June 2016. A total of 2,258 isolates were collected, of which 2,230 were from children $\leq 15$ years old and 28 from adults. The database including 2,094 clinical isolates and 164 carriage isolates mostly from healthy students in schools. Clinical isolates were primarily from pediatric patients presenting with scarlet fever $(n=1,717)$, tonsillitis $(n=355)$, invasive infections $(n=1)$, or HenochSchonlein purpura $(n=1)$. S. pyogenes isolates were confirmed by latex-agglutination with the Diagnostic Streptococcal Grouping Kit (Oxoid, Hampshire, UK) and Vitek 2 system (bioMérieux, Marcy l'Etoile, France). All 2,258 isolates were tested for fluoroquinolone resistance.

\section{Molecular Typing and Genomic Fingerprinting of $S$. pyogenes}

All isolates were characterized by $\mathrm{emm}$ type according to the standard protocol of Centers for Disease Control and Prevention (CDC; http://www.cdc.gov/streplab/protocol-emmtype.html). Pulsed-field gel electrophoresis (PFGE), a method involving chromosomal DNA macrorestriction, was performed to describe the genomic relationships among the GAS population using restriction endonuclease SmaI (TaKaRa, Dalian, China). PFGE patterns were analyzed using BioNumerics software package (version 6.5; Applied Maths, Austin, TX, USA) under the unweighted pair group method and an arithmetic averages (UPGMA) clustering algorithm, with settings as $1.0 \%$ optimization and $1.5 \%$ band tolerance (Carrico et al., 2006) As recommended in Hong Kong scarlet fever outbreak investigation (Tse et al., 2012), isolates with $80 \%$ similarity of PFGE bands were assigned to the same cluster. In the same cluster, isolates with indistinguishable PFGE pattern (no different bands) were assigned to the same clone. 


\section{FQs Susceptibility Test}

Minimum inhibitory concentration (MIC) of levofloxacin was determined and interpreted with the broth microdilution method procedure recommended by the Clinical and Laboratory Standards Institute (CLSI) guidelines published in 2015 (Clinical and Laboratory Standards Institute, 2015). According to the breakpoints in CLSI 2015, FQ susceptibility were defined as susceptible, intermediate-resistant, and resistant when the isolates with MIC to levofloxacin as $\leq 2 \mu \mathrm{g} / \mathrm{ml}, 4 \mu \mathrm{g} / \mathrm{ml}$, and $\geq 8 \mu \mathrm{g} / \mathrm{ml}$. S. pyogenes FQ non-susceptibility was defined as intermediate-resistant and resistant isolates. Efflux pump activity in the FQ non-susceptible isolates was investigated by the agar dilution method according to CLSI 2015 guidelines, comparing the MICs to levofloxacin and ciprofloxacin in the presence or absence of $30 \mu \mathrm{g} / \mathrm{ml}$ reserpine (Sigma-Aldrich), an efflux pump blocker (Jones et al., 2003). When a FQ-non-susceptible isolate showed a four-fold or greater decrease in the MIC to levofloxacin or ciprofloxacin, the efflux phenotype was deemed to be positive, which supported the involvement of the efflux mechanism (Jones et al., 2003).

\section{Sequencing of Quinolone \\ Resistance-Associated Genes}

The QRDR sequences of genes encoding the topoisomerase IV (parC and parE) and the gyrase ( $g y r A$ and gyrB) were amplified and sequenced as previously described (Jones et al., 2003). Sequences of parC (accession number AF220946: position 64561), parE (accession number AE004092: 752342-754294), gyrA (accession number AF220945: 79-540) and gyrB (accession number AE004092: 581675-583627) from the quinolonesusceptible S. pyogenes strain SF370 (ATCC 700294) were defined as reference alleles (Malhotra-Kumar et al., 2005). The genome sequences of $S$. pyogenes isolates from Hong Kong and mainland China and the QRDR sequence of parC from 2 S. pneumonia, 7 S. agalactiae, 21 S. dysgalactiae, 1 S. canis, $1 S$. iniae, 1 S. porcinus, $2 S$. equi, and 1 S. difficilis isolates were retrieved from GenBank with the accession number listed in Tables S1, S2, respectively. The Lasergene software package (version 7.1; DNASTAR, Wisconsin, USA) was used to analyze nucleotide sequences and the deduced amino acid sequences. MEGA 5 (http://www.megasoftware.net/) was used to generate multi-sequence alignments and Neighbor-joining phylogenetic analysis. Clades were determined with the bootstrap values $>75 \%$ in the bootstrap test with 1,000 replicates. The evolutionary distances were computed by the Kimura two-parameter method and are in the units of the number of base substitutions per site.

\section{Detection of Other Resistance-Associated Genes}

Genes associated with macrolide resistance (ermB, ermA, and mef) and tetracycline resistance (tet $M$ and tet $O$ ) were screened by PCR using primers and reaction parameters as previously described (Pérez-Trallero et al., 2007).

\section{Statistical Analysis}

Statistical analysis was performed using SPSS (version 20.0; IBM). Fisher's exact test was used for the comparison of FQ resistance frequency between isolates from children and adults. Statistical significance was assessed at $P<0.05$.

\section{Accession Numbers}

The sequences of the parC alleles defined in this study were submitted to GenBank under accession numbers MF278797 to MF278806, while the parE QRDR sequences of the 30 FQ-nonsusceptible isolates as MG894399 to MG894428.

\section{Ethical Aspects}

All GAS isolates characterized in this study were collected from GAS infection cases and their close contacts, as part of routine clinical management of patients and Shanghai surveillance system for scarlet fever, according to guidelines of infectious diseases used in China nationwide. Informed consent from patients and close contacts was approved by Shanghai Municipal Center for Disease Control and Prevention ethical review committee (No: 2016-4) (Chen et al., 2017).

\section{RESULTS}

\section{FQs Susceptibility of S. pyogenes}

A total of 2,258 S. pyogenes isolates were collected during 20112016 , including 2,230 isolates from children $\leq 15$ years old and 28 from adults. There were 30 (1.3\%) FQ-non-susceptible isolates; 15 resistant (MIC range $8-16 \mu \mathrm{g} / \mathrm{ml}$ ) and 15 intermediateresistant $(\mathrm{MIC}=4 \mu \mathrm{g} / \mathrm{ml})$ isolates. The 30 isolates were all from clinical patients. Frequencies of FQ-non-susceptible isolates were different between children and adults (1.2 and 14.3\%, respectively; $P<0.05)$. Among the 1,717 isolates from scarlet fever patients, 24 (1.4\%) were non-susceptible to FQs. The FQnon-susceptible isolates were discovered every year during 20112016, with the frequency fluctuating between $2.4 \%$ in 2011 and $0.9 \%$ in 2016 (Figure 1). Alarmingly, twenty-four (80\%) of the 30 FQ-non-susceptible isolates were also resistant to erythromycin $(>128 \mu \mathrm{g} / \mathrm{ml})$, clindamycin $(>128 \mu \mathrm{g} / \mathrm{ml})$, and tetracycline $(8-$ $32 \mu \mathrm{g} / \mathrm{ml}$ ) (Table 1 ), and were identified to carry the resistanceassociated genes ermB and tet $M$, while another isolate was resistant to only FQs and tetracycline $(32 \mu \mathrm{g} / \mathrm{ml})$, possessing tet $M$.

\section{Molecular Epidemiology of FQ-Non-susceptible S. pyogenes}

Molecular typing of the 2,258 isolates identified $16 \mathrm{emm}$ types, with emm 12 and emm 1 isolates constituting 60.3 and $36.1 \%$ of the isolates respectively (Table 1). The thirty FQ-non-susceptible isolates were assigned to seven emm types (Table 1), including emm 12 (1.1\%, 15/1,362), emm6 (100\%, 6/6), emm11 (100\%, 4/4), emm 89 (13.3\%, 2/15), emm 1 (0.1\%, 1/712), emm87 (100\%, $1 / 1)$, and emm $94(100 \%, 1 / 1)$. With the exception of $6 \mathrm{emm} 12$ isolates, all FQ-non-susceptible emm types were resistant to both macrolides and tetracycline (Table $\mathbf{1}$ ).

To examine the genomic relationships of the FQ-resistant population, genome fingerprinting of the $30 \mathrm{FQ}$-non-susceptible isolates by PFGE was performed. Six clusters (A-F) were observed (Figure 2). The emm6, emm 11 , and emm 89 isolates were clonal as defined by an identical PFGE pattern whereas the emm 12 family clustered into multiple subtypes. 

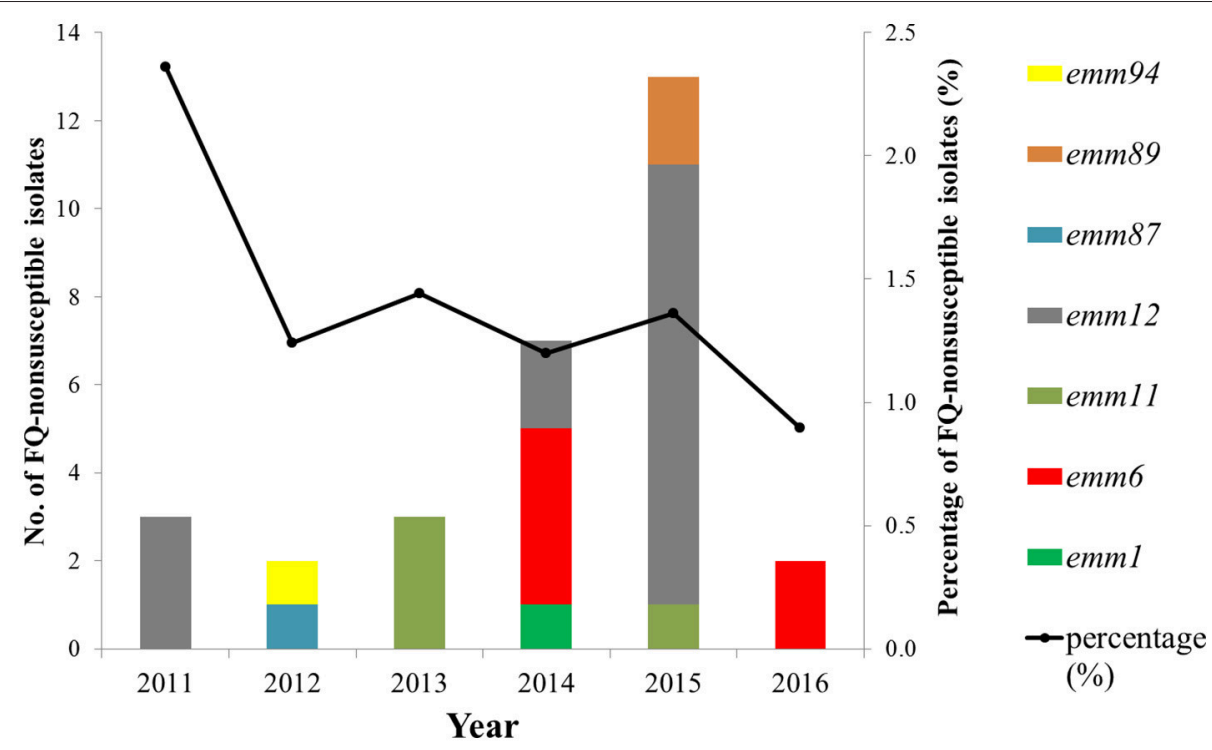

FIGURE 1 | Frequency and emm-type distribution of fluoroquinolone non-susceptibility in S. pyogenes strains isolated between 2011 to 2016 from Shanghai, China.

TABLE 1 | Antimicrobial susceptibility of isolates with different emm types.

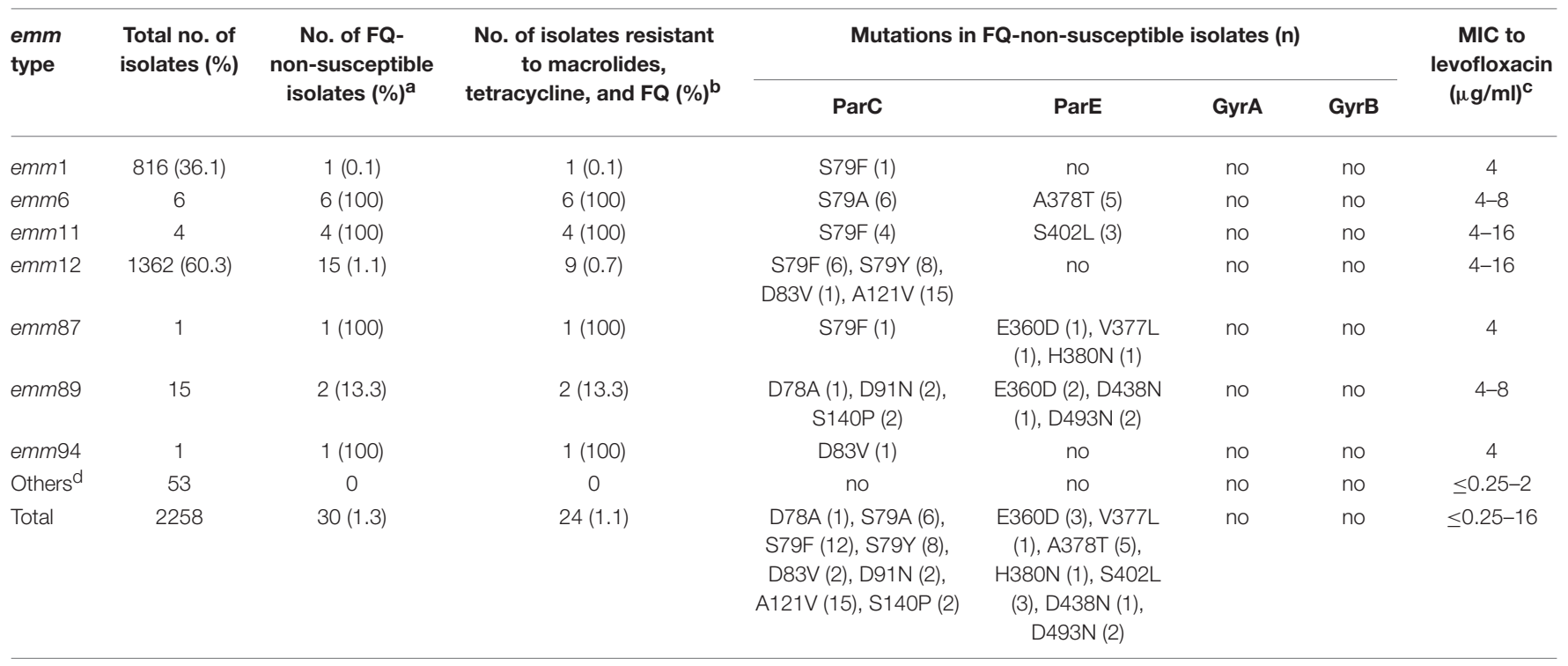

${ }^{a} F Q$, fluoroquinolone.

${ }^{b}$ Resistance to macrolides and tetracycline in all tested isolates was associated with ermB and tetM gene carriage, respectively.

${ }^{c} \mathrm{MIC}$, minimum inhibitory concentration.

dOther emm type isolates included $2 x$ emm3, 3x emm4, 12x emm5, 10x emm22, 10x emm75, 1x emm103, 13x emm170, 1x emm203, and 1x emm227 isolates.

\section{Characterization of FQ Resistance Mechanisms}

To examine the genetic basis of FQ-non-susceptibility, the parC, parE, gyrA, and $\operatorname{gyr} B$ sequences were sequenced and analyzed. Of the 30 FQ-non-susceptible isolates, all harbored at least one amino acid alteration in ParC, 11 (36.7\%) harbored ParE mutations, and none had mutations in GyrA and GyrB QRDRs (Table 2). In ParC, alteration at the amino acid site 79 (Ser) was found in 26 (86.7\%) FQ-non-susceptible isolates, including S79A (emm6), S79F (emm1, emm11, emm12, and emm87), and S79Y (emm12). The mutation A121V was found in emm12 isolates irrespective of the FQ susceptibility. Less common mutations included D78A (1), D91N (2), S140P (2), and a novel mutation, D83V (2). A total of 9 parC nucleotide alleles were found in the 30 FQ-non-susceptible isolates, designated as SHparC1- SHparC9 (Table 2). With the exception of SHparC6 that was represented by isolates of $\mathrm{emm} 1$ and emm87, each parC allele was represented by isolates of the same emm type. In ParE QRDRs, mutations 


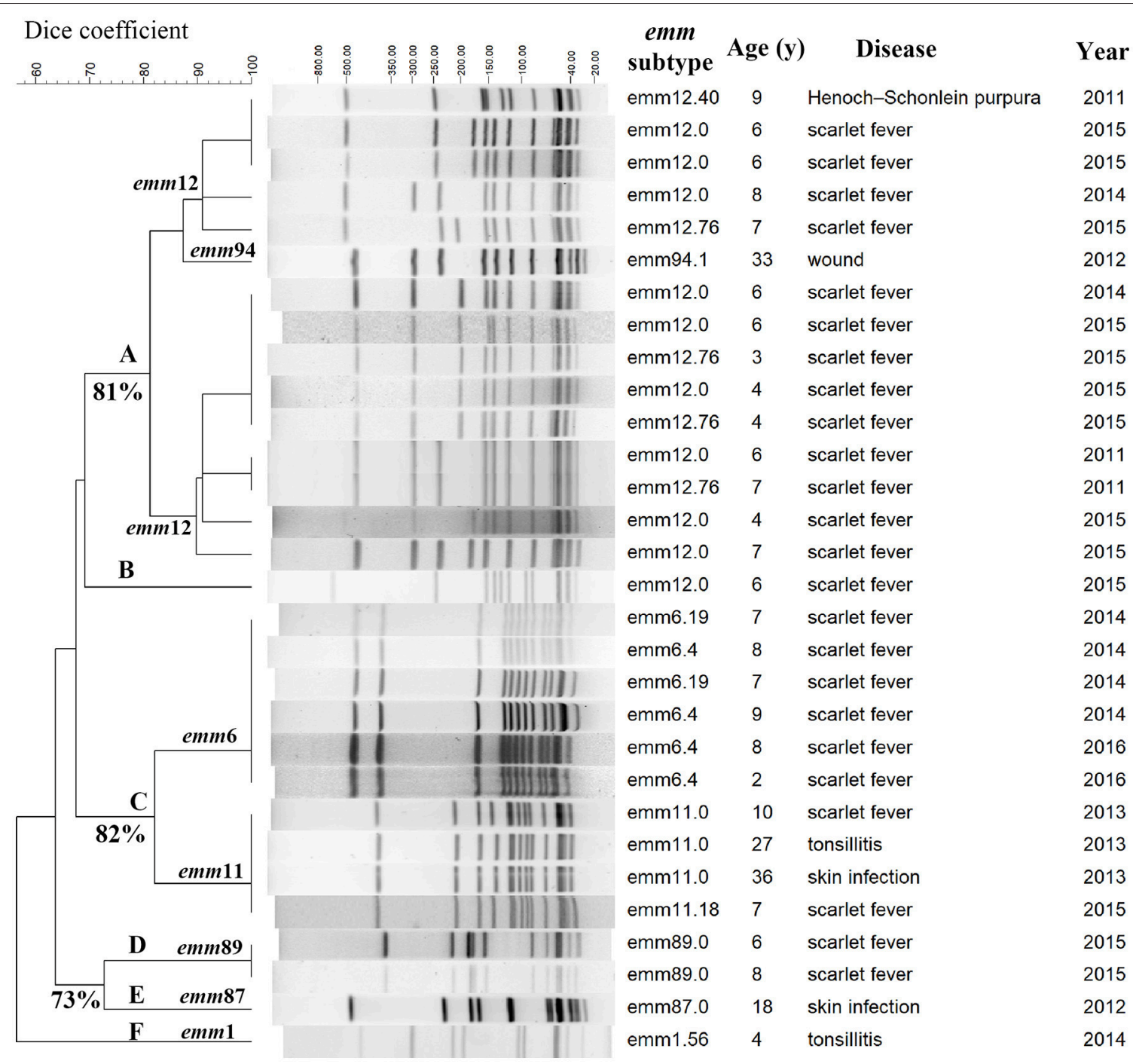

FIGURE 2 | PFGE patterns of 30 fluoroquinolone-non-susceptible S. pyogenes strains isolated between 2011 to 2016 in Shanghai, China. Clusters A-F were assigned according to $80 \%$ similarity, and, isolates with indistinguishable pulsed-field gel electrophoresis (PFGE) pattern (no different bands) were assigned to the same clone. The numbers of similarity are labeled next to the branches.

of 7 amino acid sites were identified in 11 FQ-non-susceptible isolates (Table 2), including E360D (3), V377L (1), A378T (5), H380N (1), S402L (3), D438N (1), and D493N (2). To further expand on these findings, we tested the efflux phenotype on all 30 FQ-non-susceptible isolates. None of the FQ-non-susceptible isolates showed a four-fold or greater decrease in the MIC to levofloxacin or ciprofloxacin after adding reserpine $(30 \mu \mathrm{g} / \mathrm{ml})$ indicating that the FQ tolerance could not be explained by the efflux pump mechanism.

\section{Conservation of ParC Mutations Within Whole Genome Sequences From Wider Chinese Clinical Isolates}

One hundred and seventy S. pyogenes genome sequences of isolates from Hong Kong (152) and mainland China (18) are publically available, including $136 \mathrm{emm} 12$ and $34 \mathrm{emm} 1$ strains isolated between 2004 to 2012. Examination of the QRDR genes identified 13 (7.6\%) isolates with mutations in ParC QRDR, including $12 \mathrm{emm} 12$ from Hong Kong belonging to three different evolutionary lineages and $1 \mathrm{emm} 1$ isolates from mainland China (Heilongjiang Province), and no mutations within ParE, GyrA, and GyrB. The most common ParC mutation was S79F in ParC (SHparC2) with one emm1 isolate harboring alteration D83G in ParC, which was designated as a new parC allele: SHparC10.

\section{Evolution of parC Mutations Within Streptococcaceae}

Phylogenetic analysis involving 51 QRDR parC sequences from S. pyogenes, S. pneumonia, S. agalactiae, S. dysgalactiae, S. canis, 
TABLE 2 | Nucleotide changes identified in the quinolone resistance-determining regions of different parC alleles.

\begin{tabular}{|c|c|c|c|c|c|}
\hline parC allele & $\begin{array}{l}\text { MIC to levofloxacin } \\
\qquad(\mu \mathrm{g} / \mathrm{ml})^{a}\end{array}$ & $\begin{array}{l}\text { No. of } \\
\text { isolates }\end{array}$ & emm subtype & ParC mutation & $\begin{array}{l}\text { References to ParC with the same } \\
\text { mutation pattern }\end{array}$ \\
\hline SHparC1 & $4-8$ & 8 & $12.0(6), 12.76(2)$ & $\mathrm{S} 79 \mathrm{Y}(\mathrm{TCC} \rightarrow \mathrm{TAC}) \mathrm{A} 121 \mathrm{~V}^{\mathrm{b}}(\mathrm{GCT} \rightarrow \mathrm{GTT})$ & Malhotra-Kumar et al., 2005 \\
\hline SHparC2 & $4-16$ & 6 & $12.0(3), 12.40(1), 12.76(2)$ & $\mathrm{S} 79 \mathrm{~F}(\mathrm{TCC} \rightarrow \mathrm{TTC}) \mathrm{A} 121 \mathrm{~V}(\mathrm{GCT} \rightarrow \mathrm{GTT})$ & $\begin{array}{l}\text { Richter et al., 2003; Rivera et al., } \\
\text { 2005; Lin et al., } 2015\end{array}$ \\
\hline SHparC3 & 4 & 1 & $12.0(1)$ & $\mathrm{D} 83 \mathrm{~V}(\mathrm{GAT} \rightarrow \mathrm{GTT}) \mathrm{A} 121 \mathrm{~V}(\mathrm{GCT} \rightarrow \mathrm{GTT})$ & This study \\
\hline SHparC4 & $4-16$ & 4 & $11.0(3), 11.18(1)$ & $\mathrm{S} 79 \mathrm{~F}(\mathrm{TCC} \rightarrow \mathrm{TTC})$ & $\begin{array}{l}\text { Malhotra-Kumar et al., 2005; } \\
\text { Orscheln et al., 2005; Biedenbach } \\
\text { et al., 2006; Pletz et al., 2006; } \\
\text { Montes et al., 2010; Arai et al., } 2011\end{array}$ \\
\hline SHparC5 & 4 & 1 & $94.1(1)$ & D83V $($ GAT $\rightarrow$ GTT) & This study \\
\hline SHparC6 & 4 & 2 & $1.56(1), 87.0(1)$ & $\mathrm{S79F}(\mathrm{TCC} \rightarrow \mathrm{TTC})$ & Wajima et al., 2008 \\
\hline SHparC7 & $4-8$ & 6 & $6.19(2), 6.4(4)$ & S79A (TCC $\rightarrow$ GCC) & $\begin{array}{l}\text { Albertí et al., 2005; Malhotra-Kumar } \\
\text { et al., 2005, 2009; Orscheln et al., } \\
\text { 2005; Powis et al., 2005; Rivera et al., } \\
\text { 2005; Yan et al., 2008; Smeesters } \\
\text { et al., 2009; Montes et al., 2010; Van } \\
\text { Heirstraeten et al., } 2012\end{array}$ \\
\hline SHparC8 & 4 & 1 & $89.0(1)$ & $\mathrm{D} 91 \mathrm{~N}(\mathrm{GAT} \rightarrow \mathrm{AAT}), \mathrm{S} 140 \mathrm{P}(\mathrm{TCT} \rightarrow \mathrm{CCC})$ & Malhotra-Kumar et al., 2005 \\
\hline SHparC9 & 8 & 1 & $89.0(1)$ & $\begin{array}{l}\mathrm{D} 78 \mathrm{~A}(\mathrm{GAT} \rightarrow \mathrm{GCT}), \mathrm{D} 91 \mathrm{~N}(\mathrm{GAT} \rightarrow \mathrm{AAT}) \\
\mathrm{S} 140 \mathrm{P}(\mathrm{TCT} \rightarrow \mathrm{CCC})\end{array}$ & This study \\
\hline SHparC10C & $N D^{d}$ & 1 & $1.0(1)$ & $\mathrm{D} 83 \mathrm{G}(\mathrm{GAT} \rightarrow \mathrm{GGT})$ & $\begin{array}{l}\text { Van Heirstraeten et al., 2012; Lin } \\
\text { et al., } 2015\end{array}$ \\
\hline
\end{tabular}

${ }^{a} \mathrm{MIC}$, minimum inhibitory concentration.

${ }^{b} \mathrm{~A} 121 \mathrm{~V}$ was also found in fluoroquinolone-susceptible isolates.

'Sequence was extracted from the genome of HLJGAS2022.

${ }^{d} N D$, not determined.

S. iniae, S. porcinus, S. equi, and S. difficilis was performed to examine evidence of lateral gene transfer and FQ resistance. All S. pyogenes and S. dysgalactiae isolates clustered together into a single clade (Clade S. pyogenes \& S. dysgalactiae, Figure 3). The clade can be further divided into two sub-branches: the $S$. pyogenes sub-branch and the $S$. dysgalactiae sub-branch. Most of the $S$. pyogenes parC alleles (8/10) defined in this study were grouped into $S$. pyogenes sub-branch, while alleles SHparC8 and SHparC9 were found in the $S$. dysgalactiae sub-branch. Five parC sequences of $S$. dysgalactiae, including FQ-susceptible and FQ-non-susceptible isolates, were included in the S. pyogenes sub-branch.

\section{DISCUSSION}

This study is the first report that investigates and characterizes FQ-non-susceptibility within $S$. pyogenes from mainland China during 2011-2016, the period overlapping an ongoing multidrug resistant GAS scarlet fever epidemic in China (Tse et al., 2012; Yang et al., 2013; Davies et al., 2015). We identified FQ nonsusceptibility to be $1.3 \%$ in 2,258 GAS isolates from Shanghai, China, including within emm12 and emm 1 scarlet fever clones resistant to macrolides and tetracycline. The appearance of these clones has further narrowed the choices of treatment for GAS infections. We observed a significant difference in FQ resistance frequencies in GAS isolated from adults (14.3\%) compared to children (1.2\%). This may in part be attributable to clinical practice where administration of fluoroquinolones is largely restricted to adults due to cartilage toxicity observed in children (Stahlmann et al., 1990).
Since 2006, very high frequencies (>90\%) of resistance to macrolide and tetracycline in GAS isolates have been reported in mainland China (Jing et al., 2006; Chen et al., 2012; Yang et al., 2013). Coincidently, fluoroquinolone consumption has increased over the last two decades and is currently the third most consumed antibiotic in China (Van Boeckel et al., 2014). Our study identified that $80 \%$ of FQ-non-susceptible isolates were also resistant to both macrolides $(\mathrm{erm} B)$ and tetracycline (tet $M)$. In previous GAS studies, only three isolates harboring this multidrug resistance profile have been reported in a Belgian study (Malhotra-Kumar et al., 2005). With the emergence of antimicrobial resistance in respiratory pathogens intrinsically linked with antibiotic consumption (Goossens et al., 2005), the appearance of multidrug resistant clones through selective pressure is highly probable.

Compared with the prevalence of GAS FQ-non-susceptibility in Taiwan (11.1\% in 2005-2012), Japan (14.1\% in 2010-2012), Belgium (11.4\% in 2007-2010), Spain (13\% in 2005-2007), and USA (9\% in 2003) (Yan et al., 2008; Montes et al., 2010; Van Heirstraeten et al., 2012; Wajima et al., 2013; Lin et al., 2015), the overall prevalence of FQ-non-susceptible S. pyogenes in Shanghai (1.3\%) and Beijing (3.4\%) (Yang et al., 2013) are relatively low. This may be attributed to the limited administration of FQ to pediatric patients in China, which constitute most of isolates in this study (98.8\%) and all the isolates in the Beijing study. Correspondingly, low frequencies of FQ resistance was found in the most frequently isolated GAS emm type in this study, emm 12 (1.1\%). The frequencies of FQ resistance in emm 12 isolates varies in different countries, from $0(0 / 45)$ in Spain to $5 \%(1 / 20)$ in Japan, and to 50\% (33/66) in Taiwan (Montes et al., 2010; Wajima 


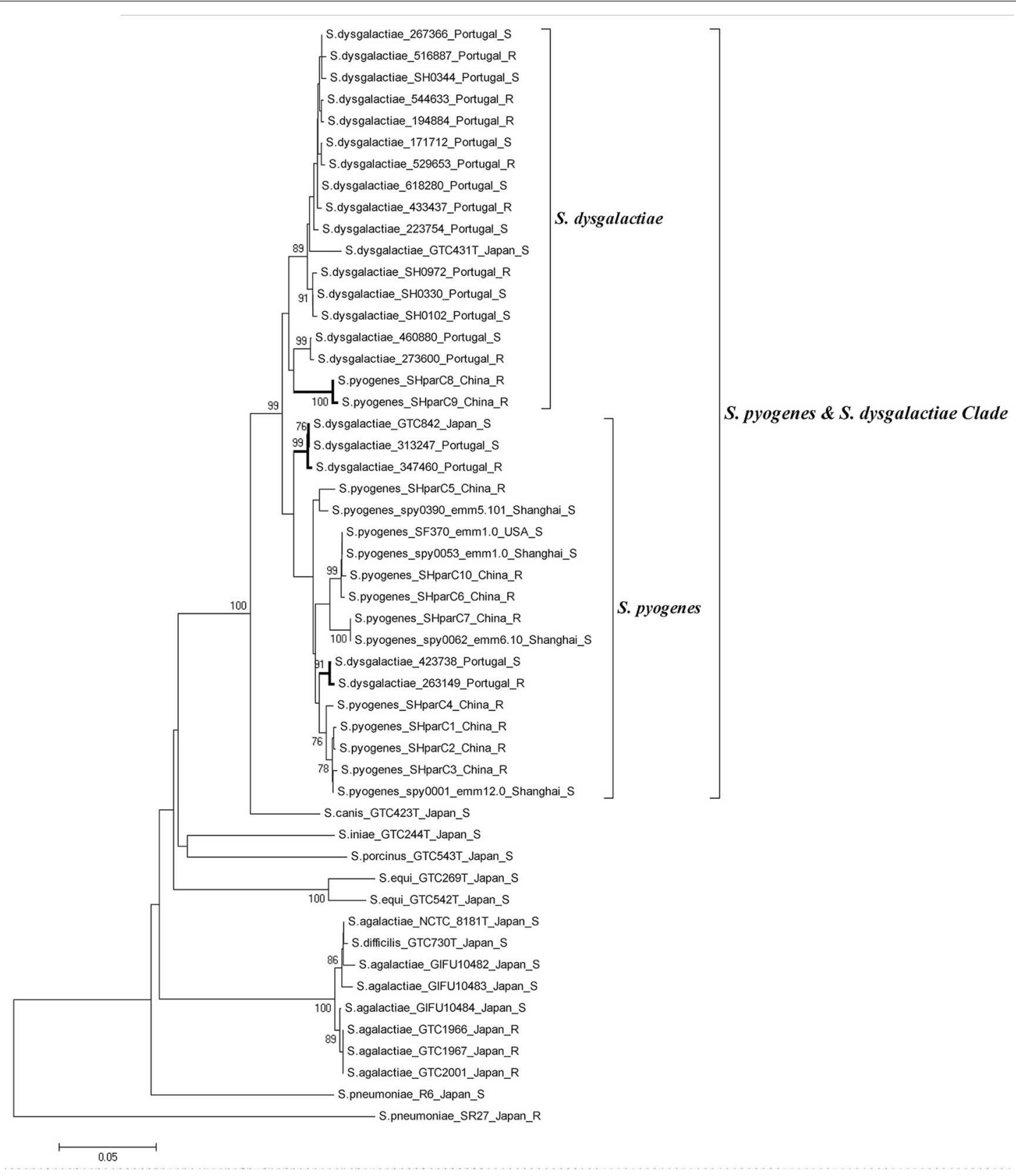

FIGURE 3 | Phylogenetic analysis of parC quinolone-resistance-determining region from different streptococcal species. Strains are shown as; species, strain number or parC allele; (emm type); country or district; and fluoroquinolone susceptibility. All fluoroquinolone-non-susceptible isolates were labeled with "R." Phylogenetic analysis was conducted in MEGA 5 using Neighbor-joining. Bootstrap values are indicated when support is >75\% (from 1,000 replicates). The tree is drawn to scale, with the branch lengths being in the same units as those of the evolutionary distances used to infer the phylogenetic tree. The evolutionary distances were computed by the Kimura two-parameter method and are in the units of the number of base substitutions per site.

et al., 2013; Lin et al., 2015). Based on whole genome analysis of scarlet fever isolates in Hong Kong (Davies et al., 2015), we estimate the frequency of emm 12 FQ resistance in Hong Kong was $9.0 \%(12 / 134)$, of which $66.7 \%(8 / 12)$ were also resistant to macrolide and tetracycline possessing the corresponding molecular markers ermB and tet $M$ (Table S1); however, validation of these findings through FQ susceptibility testing is required to confirm this genetic observation. The emergence of FQ resistance within ongoing scarlet fever epidemics in Hong Kong and mainland China (Luk et al., 2012; Yang et al., 2013) calls for more precaution when prescribing fluoroquinolones for suspected GAS infections.

In this study, FQ-non-susceptible emm6 isolates were discovered in China for the first time. Orscheln et al. proposed that FQ resistance is an intrinsic property of the emm6 lineage, with mutations in ParC driving FQs resistance long before 
the introduction of FQ antibiotics (Orscheln et al., 2005). The global dissemination of emm 6 strains was suggested to be the main reason for the increased proportion of FQ-non-susceptible S. pyogenes in Belgium and Spain (Montes et al., 2010; Van Heirstraeten et al., 2012). All six emm6 isolates identified in this study were a single FQ-non-susceptible clone on the basis of PFGE, and harbored the same ParC QRDR S79A mutation (parC allele of SHparC7) with those emm6 isolates from USA and Spain (Orscheln et al., 2005; Rivera et al., 2005). In this study, the emm 6 clone was also resistant to erythromycin, clindamycin, and tetracycline. This observation is mirrored for the global emm 11 clone (Wajima et al., 2008, 2013; Van Heirstraeten et al., 2012) with all four emm11 GAS isolated in this study representing a single FQ-non-susceptible clone harboring an S79F ParC mutation and the macrolide and tetracycline resistance markers. Collectively, monitoring for the global dissemination of these multidrug resistant GAS clones is warranted.

All FQ-non-susceptible S. pyogenes in this study showed a lowlevel resistance (MIC range, $4-16 \mu \mathrm{g} / \mathrm{ml}$ ) and molecular analyses of the classic FQ-non-susceptibility genetic markers suggests that FQ-non-susceptibility in China is primarily linked to ParC QRDR mutations, for they all harbored ParC mutation(s), occasionally with ParE mutation(s), and none with GyrA and GyrB mutations or involvement of efflux pump, in addition, isolates with ParE mutation(s) showed similar MIC values to levofloxacin and ciprofloxacin compared to those only possessing ParC mutation(s). This supports the stepwise theory of FQ resistance: without GyrA mutation, where mutations in ParC only confer a low-level resistance (Billal et al., 2007). The most common alterations occurred in the amino acid site Ser79, a potential site to bind FQs (Laponogov et al., 2007), which is supported by findings from American and European countries (Orscheln et al., 2005; Smeesters et al., 2009). Polymorphisms of parC QRDR were found in FQ-non-susceptible isolates, and they were mostly linked with $\mathrm{emm}$ types in this study. This suggested most of the mutations might occur spontaneously within the strains' evolutionary process, which was validated by the phylogenetic analysis of the parC QRDR however two parC alleles may have arisen through horizontal gene transfer from other streptococcal species such as $S$. dysgalactiae. Some parC sequence of FQ-susceptible and FQ-non-susceptible $S$. dysgalactiae were also grouped into the $S$. pyogenes sub-branch in this study, which provided more evidence for a global gene pool shared by $S$. dysgalactiae and $S$. pyogenes (Pinho et al., 2010). A number of ParE mutations were identified in this study, including three mutations of E360D, V377L, and H380N concurrently present in a emm87 isolate and an A378T mutation identified only in emm6 isolates as documented previously (Malhotra-Kumar et al., 2005; Lin et al., 2015), while S402L mutation was only found in emm 11 isolates, and mutations of D438N and D493N were only identified in emm89 isolates, which were first reported in this study. These phenomena suggest most of the ParE mutations might be evolutionary linked with $\mathrm{emm}$ type rather than drivers of FQ resistance.

One limitation of this study is the small sample size of isolates from adults, which might lead to a possible bias when comparing the FQ-non-susceptibility frequency between isolates from children and adults, but the appearance of adult FQ-nonsusceptible isolates in different years (2012 and 2013) supported, to some extent, the validation of the high frequency in adults. The lack of adult isolates can largely be attributed to the fact that only scarlet fever, which is primarily a disease of children, is the only notifiable disease among all the GAS infections in China (Yang et al., 2013). Another possible attribution might be that the morbidity of GAS infections is low in adults; however, more data on adult GAS infections in China is required to support this hypothesis (Chen et al., 2017). Our finding of a higher rate of FQ-non-susceptibility in isolates from adults (14.3\%) is alarming and warrants further investigation at a population level.

In conclusion, the emergence of macrolide, tetracycline and fluoroquinolone- non-susceptible $S$. pyogenes in Shanghai, China during the period of scarlet fever epidemics was revealed in this study. Although FQ resistance was infrequent in mainland China, the presence of macrolide, tetracycline and fluoroquinolone multidrug-resistant clones across multiple $S$. pyogenes emm sequence types is alarming and the spread of these isolates should be monitored globally.

\section{AUTHOR CONTRIBUTIONS}

MC and MZ: conceived and designed the experiments; YS, JC, and CZ: performed all experiments; YS, JC, and MD: analyzed the data; YS, KG, DQ, HJ, WY, and YL: collected clinical specimens; MC and MZ: supervised the study and wrote the paper; MD: revised the paper. All authors have read and approved the final manuscript.

\section{FUNDING}

This study was supported by grants from National Natural Science Foundation of China (81601801), Shanghai RisingStar Program (17QA1403100), a Municipal Human Resources Development Program for Outstanding Young Talents in Medical and Health Sciences in Shanghai (2017YQ039), Natural Science Foundation of Shanghai (16ZR1433300), and Key project funded by Shanghai Municipal Commission of Health and Family Planning (201640002). MD is supported by the National Health and Medical Research Council of Australia (NHMRC) grants 1126805 and 1130455 . The funders had no role in study design, data collection and interpretation, or the decision to submit the work for publication.

\section{ACKNOWLEDGMENTS}

We thank James E. Bray for assembling the S. pyogenes genomes.

\section{SUPPLEMENTARY MATERIAL}

The Supplementary Material for this article can be found online at: https://www.frontiersin.org/articles/10.3389/fmicb. 2018.00542/full\#supplementary-material 


\section{REFERENCES}

Albertí, S., Cortés, G., García-Rey, C., Rubio, C., Baquero, F., GarcíaRodríguez, J. A. et al. (2005). Streptococcus pyogenes pharyngeal isolates with reduced susceptibility to ciprofloxacin in Spain: mechanisms of resistance and clonal diversity. Antimicrob. Agents Chemother. 49, 418-420. doi: 10.1128/AAC.49.1.418-420.2005

Arai, K., Hirakata, Y., Yano, H., Kanamori, H., Endo, S., Hirotani, A., et al. (2011). Emergence of fluoroquinolone-resistant Streptococcus pyogenes in Japan by a point mutation leading to a new amino acid substitution. J. Antimicrob. Chemother. 66, 494-498. doi: 10.1093/jac/dkq477

Biedenbach, D. J., Toleman, M. A., Walsh, T. R., and Jones, R. N. (2006). Characterization of fluoroquinolone-resistant beta-hemolytic Streptococcus spp. isolated in North America and Europe including the first report of fluoroquinolone-resistant Streptococcus dysgalactiae subspecies equisimilis: report from the SENTRY Antimicrobial Surveillance Program (1997-2004). Diagn. Microbiol. Infect. Dis. 55, 119-127. doi: 10.1016/j.diagmicrobio.2005.12.006

Billal, D. S., Fedorko, D. P., Yan, S. S., Hotomi, M., Fujihara, K., Nelson, N., et al. (2007). In vitro induction and selection of fluoroquinolone-resistant mutants of Streptococcus pyogenes strains with multiple emm types. J. Antimicrob. Chemother. 59, 28-34. doi: 10.1093/jac/dkl428

Carapetis, J. R., Steer, A. C., Mulholland, E. K., and Weber, M. (2005). The global burden of group A streptococcal diseases. Lancet Infect. Dis. 5, 685-694. doi: 10.1016/S1473-3099(05)70267-X

Carrico, J. A., Silva-Costa, C., Melo-Cristino, J., Pinto, F. R., de Lencastre, H., Almeida, J. S., et al. (2006). Illustration of a common framework for relating multiple typing methods by application to macrolide-resistant Streptococcus pyogenes. J. Clin. Microbiol. 244, 2524-2532. doi: 10.1128/JCM.02536-05

Chalker, V., Jironkin, A., Coelho, J., Al-Shahib, A., Platt, S., Kapatai, G., et al. (2017). Genome analysis following a national increase in Scarlet Fever in England 2014. BMC Genomics 18:224. doi: 10.1186/s12864-017-3603-Z

Chen, M., Wang, W., Tu, L., Zheng, Y., Pan, H., Wang, G., et al. (2017). An emm5 Group A Streptococcal outbreak among workers in a factory manufacturing telephone accessories. Front. Microbiol. 8:1156. doi: 10.3389/fmicb.2017.01156

Chen, M., Yao, W., Wang, X., Li, Y., Wang, G., Zhang, X., et al. (2012). Outbreak of scarlet fever associated with emm12 Type group A Streptococcus in 2011 in Shanghai, China. Pediatr. Infect. Dis. J. 31, e158-e162. doi: 10.1097/INF.0b013e31825874f3

Clinical and Laboratory Standards Institute (2015). Performance Standards for Antimicrobial Susceptibility Testing: Twenty-Fifth Informational Supplement, CLSI Document M100-S25. (Wayne, PA: CLSI).

Davies, M. R., Holden, M. T., Coupland, P., Chen, J. H., Venturini, C., Barnett, T. C., et al. (2015). Emergence of scarlet fever Streptococcus pyogenes emm 12 clones in Hong Kong is associated with toxin acquisition and multidrug resistance. Nat. Genet. 47, 84-87. doi: 10.1038/ng.3147

Duesberg, C. B., Malhotra-Kumar, S., Goossens, H., McGee, L., Klugman, K. P., Welte, T., et al. (2008). Interspecies recombination occurs frequently in quinolone resistance-determining regions of clinical isolates of Streptococcus pyogenes. Antimicrob. Agents Chemother. 52, 4191-4193. doi: 10.1128/AAC.00518-08

Goossens, H., Ferech, M., Vander Stichele, R., Elseviers, M., and Group, E. P. (2005). Outpatient antibiotic use in Europe and association with resistance: a cross-national database study. Lancet 365, 579-587. doi: 10.1016/S0140-6736(05)70799-6

Hooper, D. C. (2002). Fluoroquinolone resistance among Gram-positive cocci. Lancet Infect. Dis. 2, 530-538. doi: 10.1016/S1473-3099(02)00369-9

Jing, H. B., Ning, B. A., Hao, H. J., Zheng, Y. L., Chang, D., Jiang, W., et al. (2006). Epidemiological analysis of group A streptococci recovered from patients in China. J. Med. Microbiol. 55(Pt 8), 1101-1107. doi: 10.1099/jmm.0.46243-0

Jones, H. E., Brenwald, N. P., Owen, K. A., and Gill, M. J. (2003). A multidrug efflux phenotype mutant of Streptococcus pyogenes. J. Antimicrob. Chemother. 51, 707-710. doi: $10.1093 / \mathrm{jac} / \mathrm{dkg} 121$

Laponogov, I., Veselkov, D. A., Sohi, M. K., Pan, X. S., Achari, A., Yang, C., et al. (2007). Breakage-reunion domain of Streptococcus pneumoniae topoisomerase IV: crystal structure of a gram-positive quinolone target. PLOS ONE 2:e301. doi: 10.1371/journal.pone.0000301
Lau, E. H., Nishiura, H., Cowling, B. J., Ip, D. K., and Wu, J. T. (2012). Scarlet fever outbreak, Hong Kong, 2011. Emerging Infect. Dis. 18, 1700-1702. doi: 10.3201/eid1810.120062

Lin, J. N., Chang, L. L., Lai, C. H., Huang, Y. H., Chen, W. F., Yang, C. H., et al. (2015). High prevalence of fluoroquinolone-nonsusceptible Streptococcus pyogenes emm12 in Taiwan. Diagn. Microbiol. Infect. Dis. 83, 187-192. doi: 10.1016/j.diagmicrobio.2015.06.018

Luk, E. Y., Lo, J. Y., Li, A. Z., Lau, M. C., Cheung, T. K., Wong, A. Y., et al. (2012). Scarlet fever epidemic, Hong Kong, 2011. Emerging Infect. Dis. 18, 1658-1661. doi: 10.3201/eid1810.111900

Malhotra-Kumar, S., Lammens, C., Chapelle, S., Mallentjer, C., Weyler, J., and Goossens, H. (2005). Clonal spread of fluoroquinolone nonsusceptible Streptococcus pyogenes. J. Antimicrob. Chemother. 55, 320-325. doi: 10.1093/jac/dki011

Malhotra-Kumar, S., Van Heirstraeten, L., Lammens, C., Chapelle, S., and Goossens, H. (2009). Emergence of high-level fluoroquinolone resistance in emm6 Streptococcus pyogenes and in vitro resistance selection with ciprofloxacin, levofloxacin and moxifloxacin. J. Antimicrob. Chemother. 63, 886-894. doi: 10.1093/jac/dkp057

Montes, M., Tamayo, E., Orden, B., Larruskain, J., and Perez-Trallero, E. (2010). Prevalence and clonal characterization of Streptococcus pyogenes clinical isolates with reduced fluoroquinolone susceptibility in Spain. Antimicrob. Agents Chemother. 54, 93-97. doi: 10.1128/AAC.00780-09

Orscheln, R. C., Johnson, D. R., Olson, S. M., Presti, R. M., Martin, J. M., Kaplan, E. L., et al. (2005). Intrinsic reduced susceptibility of serotype 6 Streptococcus pyogenes to fluoroquinolone antibiotics. J. Infect. Dis. 191, 1272-1279. doi: $10.1086 / 428856$

Pérez-Trallero, E., Montes, M., Orden, B., Tamayo, E., García-Arenzana, J. M., and Marimón, J. M. (2007). Phenotypic and genotypic characterization of Streptococcus pyogenes isolates displaying the MLSB phenotype of macrolide resistance in Spain, 1999 to 2005. Antimicrob. Agents Chemother. 51, 1228-1233. doi: 10.1128/AAC.01054-06

Petrelli, D., Di Luca, M. C., Prenna, M., Bernaschi, P., Repetto, A., and Vitali, L. A. (2014). Characterization of levofloxacin non-susceptible clinical Streptococcus pyogenes isolated in the central part of Italy. Eur. J. Clin. Microbiol. Infect. Dis. 33, 241-244. doi: 10.1007/s10096-013-1950-5

Pinho, M. D., Melo-Cristino, J., and Ramirez, M. (2010). Fluoroquinolone resistance in Streptococcus dysgalactiae subsp. equisimilis and evidence for a shared global gene pool with Streptococcus pyogenes. Antimicrob. Agents Chemother. 54, 1769-1777. doi: 10.1128/AAC.01377-09

Pires, R., Ardanuy, C., Rolo, D., Morais, A., Brito-Avô, A., Gonçalo-Marques, J., et al. (2010). Emergence of ciprofloxacin-nonsusceptible Streptococcus pyogenes isolates from healthy children and pediatric patients in Portugal. Antimicrob. Agents Chemother. 54, 2677-2680. doi: 10.1128/AAC.01536-09

Pletz, M. W., McGee, L., Van Beneden, C. A., Petit, S., Bardsley, M., Barlow, M., et al. (2006). Fluoroquinolone resistance in invasive Streptococcus pyogenes isolates due to spontaneous mutation and horizontal gene transfer. Antimicrob. Agents Chemother. 50, 943-948. doi: 10.1128/AAC.50.3.943-948.2006

Powis, J., McGeer, A., Duncan, C., Goren, R., de Azavedo, J. C., Bast, D. J., et al. (2005). Prevalence and characterization of invasive isolates of Streptococcus pyogenes with reduced susceptibility to fluoroquinolones. Antimicrob. Agents Chemother. 49, 2130-2132. doi: 10.1128/AAC.49.5.2130-2132.2005

Reinert, R. R., Lütticken, R., and Al-Lahham, A. (2004). Highlevel fluoroquinolone resistance in a clinical Streptoccoccus pyogenes isolate in Germany. Clin. Microbiol. Infect. 10, 659-662. doi: 10.1111/j.1469-0691.2004.00890.x

Richter, S. S., Diekema, D. J., Heilmann, K. P., Almer, L. S., Shortridge, V. D., Zeitler, R., et al. (2003). Fluoroquinolone resistance in Streptococcus pyogenes. Clin. Infect. Dis. 36, 380-383. doi: 10.1086/345904

Rivera, A., Rebollo, M., Sánchez, F., Navarro, F., Miró, E., Mirelis, B., et al. (2005). Characterisation of fluoroquinolone-resistant clinical isolates of Streptococcus pyogenes in Barcelona, Spain. Clin. Microbiol. Infect. 11, 759-761. doi: 10.1111/j.1469-0691.2005.01216.x

Smeesters, P. R., Vergison, A., Junior, D. C., and Van Melderen, L. (2009). Emerging fluoroquinolone-non-susceptible group A Streptococci in two different paediatric populations. Int. J. Antimicrob. Agents 34, 44-49. doi: 10.1016/j.ijantimicag.2009.01.012 
Stahlmann, R., Merker, H. J., Hinz, N., Chahoud, I., Webb, J., Heger, W., et al. (1990). Ofloxacin in juvenile non-human primates and rats. Arthropathia and drug plasma concentrations. Arch. Toxicol. 64, 193-204. doi: $10.1007 / \mathrm{BF} 02010725$

Tse, H., Bao, J. Y., Davies, M. R., Maamary, P., Tsoi, H. W., Tong, A. H., et al. (2012). Molecular characterization of the 2011 Hong Kong scarlet Fever outbreak. J. Infect. Dis. 206, 341-351. doi: 10.1093/infdis/jis362

Van Boeckel, T. P., Gandra, S., Ashok, A., Caudron, Q., Grenfell, B. T., Levin, S. A., et al. (2014). Global antibiotic consumption 2000 to 2010: an analysis of national pharmaceutical sales data. Lancet Infect. Dis. 14, 742-750. doi: 10.1016/S1473-3099(14)70780-7

Van Heirstraeten, L., Leten, G., Lammens, C., Goossens, H., and Malhotra-Kumar, S. (2012). Increase in fluoroquinolone non-susceptibility among clinical Streptococcus pyogenes in Belgium during 2007-10. J. Antimicrob. Chemother. 67, 2602-2605. doi: 10.1093/jac/dks281

Wajima, T., Morozumi, M., Chiba, N., Shouji, M., Iwata, S., Sakata, H., et al. (2013). Associations of macrolide and fluoroquinolone resistance with molecular typing in Streptococcus pyogenes from invasive infections, 2010-2012. Int. J. Antimicrob. Agents 42, 447-449. doi: 10.1016/j.ijantimicag.2013.06.022

Wajima, T., Murayama, S. Y., Sunaoshi, K., Nakayama, E., Sunakawa, K., and Ubukata, K. (2008). Distribution of emm type and antibiotic susceptibility of group A streptococci causing invasive and noninvasive disease. J. Med. Microbiol. 57(Pt 11), 1383-1388. doi: 10.1099/jmm.0.2008/ 002642-0

Walker, M. J., Barnett, T. C., McArthur, J. D., Cole, J. N., Gillen, C. M., Henningham, A., et al. (2014). Disease manifestations and pathogenic mechanisms of Group A Streptococcus. Clin. Microbiol. Rev. 27, 264-301. doi: 10.1128/CMR.00101-13

Yan, S. S., Fox, M. L., Holland, S. M., Stock, F., Gill, V. J., and Fedorko, D. P. (2000). Resistance to multiple fluoroquinolones in a clinical isolate of Streptococcus pyogenes: identification of gyrA and parC and specification of point mutations associated with resistance. Antimicrob. Agents Chemother. 44, 3196-3198. doi: 10.1128/AAC.44.11.3196-3198.2000

Yan, S. S., Schreckenberger, P. C., Zheng, X., Nelson, N. A., Harrington, S. M., Tjhio, J., et al. (2008). An intrinsic pattern of reduced susceptibility to fluoroquinolones in pediatric isolates of Streptococcus pyogenes. Diagn. Microbiol. Infect. Dis. 62, 205-209. doi: 10.1016/j.diagmicrobio.2008.04.018

Yang, P., Peng, X., Zhang, D., Wu, S., Liu, Y., Cui, S., et al. (2013). Characteristics of group A Streptococcus strains circulating during scarlet fever epidemic, Beijing, China, 2011. Emerging Infect. Dis. 19, 909-915. doi: 10.3201/eid1906.121020

Conflict of Interest Statement: The authors declare that the research was conducted in the absence of any commercial or financial relationships that could be construed as a potential conflict of interest.

Copyright (๑) 2018 Shen, Cai, Davies, Zhang, Gao, Qiao, Jiang, Yao, Li, Zeng and Chen. This is an open-access article distributed under the terms of the Creative Commons Attribution License (CC BY). The use, distribution or reproduction in other forums is permitted, provided the original author(s) and the copyright owner are credited and that the original publication in this journal is cited, in accordance with accepted academic practice. No use, distribution or reproduction is permitted which does not comply with these terms. 